\title{
A 35-Year-Old Man Presenting Sleep-Related Painful Erections (Erpes): A Case Report and Review of Literature
}

\author{
Daniel de Freitas G. Soares, Ernani Luis Rhoden \\ Federal University of Health Sciences (UFCSPA), Porto Alegre, Brazil \\ Email:_daniel_fgs@hotmail.com, ernanirhoden@yahoo.com.br
}

Received June 14, 2013; revised July 14, 2013; accepted July 21, 2013

Copyright (C) 2014 Daniel de Freitas G. Soares, Ernani Luis Rhoden. This is an open access article distributed under the Creative Commons Attribution License, which permits unrestricted use, distribution, and reproduction in any medium, provided the original work is properly cited. In accordance of the Creative Commons Attribution License all Copyrights (C) 2014 are reserved for SCIRP and the owner of the intellectual property Daniel de Freitas G. Soares, Ernani Luis Rhoden. All Copyright (C) 2014 are guarded by law and by SCIRP as a guardian.

\begin{abstract}
Introduction: Erectile episodes occurring in the night time are considered normal and are usually related to the REM sleep. Spontaneous painful erections are unusual but they can have a great impact in the patient's quality of sleep and, for consequence, quality of life. Report: We present a patient who has been presenting painful erections which wake him up almost every night. We discuss the workup and treatment offered to the patient, as well as the short-time response and two months follow-up. Discussion: Although studies still do not explain this relationship, nocturnal erections occur only during rapid eye movement (REM) sleep, which can be confirmed by polysomnography accompanied by Nocturnal Penile Tumescence testing or RigiScan test. However, diagnosis can be established based exclusively on clinical aspects. Based on all literature reviewed, the initial treatment should safely consist in improvement in sleep architecture and pelvis muscles relaxation. Conclusion: After the first suspicion, polysomnography with rigidity measurements of nocturnal erections should be considered although clinical diagnosis and therapeutic test may be acceptable. The management we suggest is usually effective, well tolerated and sustained.
\end{abstract}

\section{KEYWORDS}

\section{Painful Erection; Priapism; Nocturnal Erections; REM-Sleep}

\section{Introduction}

Erectile episodes occurring in the night time are considered normal and are usually related to the REM sleep. Spontaneous painful erections are unusual but they can have a great impact in the patient's quality of sleep and, for consequence, quality of life. In this article we present a patient who has been presenting painful erections which wake him up almost every night.

\section{Report}

A 35-year-old Caucasian man was referred to the urologist with a complaint of intermittent nocturnal painful erections, with no other accompanying symptoms and not accompanied by sexual desire. He reported a 6 month history of repeated awakenings caused by the erections with increasing number of episodes per night and increasing frequency per week. Ultimately it had been oc- curring about three to four times per night, every night. The episodes were not related to sexual stimulation. The patient was very bothered, once the daytime fatigue and tension had indeed a great impact on his capacity for work and quality of life. On the other hand, he was satisfied with his sexual relationship, reporting a mean of four intercourses per week, all of them with normal duration painless erections. There was no past of priapism as well as ejaculation related problems. He had been treated for reactive arthritis (formerly Reiter syndrome) with doxiciclin $100 \mathrm{mg}$ twice a day for 6 weeks one year before. He has no other health problem and denies medications or illicit drugs use. No potential trigger could be identified in the history.

The urological physical examination, including digital rectal examination and detailed inspection and palpation of the glans, corpora cavernosa, anterior urethra and prepucial and penile shaft skin, was normal. A simplified 
neurological examination showed no abnormalities.

Fresh prostatic secretion examination presented 6 leukocytes per high power field.

Investigation for Clamydiatrachomatis (immunofluorescence and PCR) was negative, as it also was for herpes simplex. Blood and urine analyses showed no evidence of infection. Other laboratories exams were normal and are listed below. Serum total testosterone was 358 $\mathrm{ng} / \mathrm{mL}$ (N: $300-1000 \mathrm{ng} / \mathrm{mL}$ ), luteinizing hormone was 3.1 UI/L (N; 1.7 - $8.6 \mathrm{UI} / \mathrm{L})$, follicle-stimulating hormone was $2.9 \mathrm{UI} / \mathrm{L}(\mathrm{N} ; 1.5$ - $12.4 \mathrm{UI} / \mathrm{L})$, prolactin was 9 $\mathrm{ng} / \mathrm{mL}(\mathrm{N} ; 4.0$ - $15.2 \mathrm{ng} / \mathrm{mL})$, thyroid-stimulating hormone was $2.42 \mathrm{UI} / \mathrm{L}(\mathrm{N}: 0.3-4.2 \mathrm{UI} / \mathrm{L}$ ) and Thyroxin was $1.5 \mathrm{ug} / \mathrm{dL}$ (n; $0.7-1.9 \mathrm{ug} / \mathrm{dL}$ ). Lipids, glycemia and urinary analysis were all normal.

Also abdominal and pelvic computed tomography (CT) showed no abnormalities, while spinal Magnetic Resonance Imaging (MRI) evidenced residual signs of soronegativespondiloarthropathy.

After the initial workup, the patient started baclofen 10 $\mathrm{mg}$ and clonazepan $0.5 \mathrm{mg}$ orally at bedtime, with immediate complete response. The treatment was well tolerated and no sleepiness, loss of concentration and decrease of libido were reported. There were no symptoms relapses in 2 months of follow up.

\section{Discussion}

Based in the case presented above we did the diagnosis of sleep-related painful erection in this young individual. Clinical history and the absence of abnormalities on physical examinations as well as in the complementary evaluation was in our understanding enough to establish this diagnosis. Also the treatment approach was based in the understanding of sleep related physiology. It is well documented that in the in early adolescence, nonsexual nocturnal erections are a naturally occurring phenomenon in all healthy males, and do not have necessarily any association with dream content. They tend to occur three to four times nightly and may go unnoticed by sleeping men. However most men will be aware of an erection when they arise in the morning, creating the incorrect impression that they are a reflex response to a full bladder. [1]

Despite studies still do not explain this relationship, nocturnal erections occur only during rapid eye movement (REM) sleep, which can be confirmed by polysomnography accompanied by Nocturnal Penile Tumescence testing or RigiScan test (Figure 1). This sleep state is characterized by decrease of voluntary muscle tonus, hypotension and vasodylation. [2]

Two general states are normally described to understand sleep characteristics: rapid eye movement sleep (REM) and non-rapid eye movement sleep (NREM). NREM sleep is further subdivided into four stages. Sleep

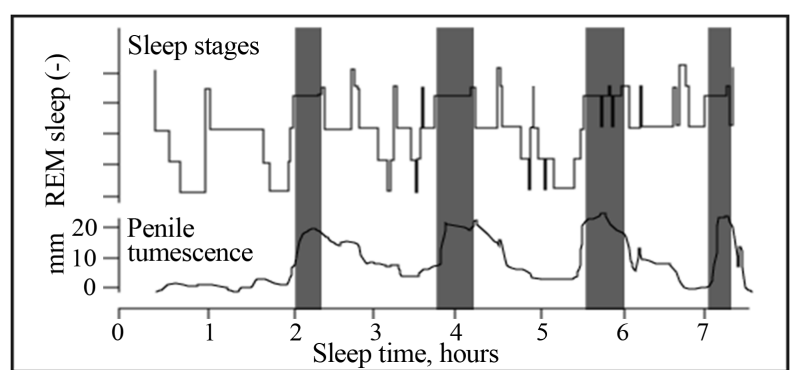

Figure 1. Penile tumescence during the different stages of sleep. Nocturnal erections occurred during periods of rapid eye movement (REM) sleep (green columns). Reprinted by permission from Karacan, I, Williams, RL, Thornby, JI, Thalis, PJ, Am J Psychiatry 1975; 132:932.

stages occur in cycles lasting 90 to 120 minutes each and four to five such cycles occur during a typical night of sleep. During the first half of the night, the individual typically passes from wakefulness briefly into stage N1 sleep and then to stages N2 and N3. Stages N2 and N3 reappear, after which REM sleep is observed for the first time. During the second half of the night, stage N2 and REM sleep alternate [3].

One of the first hypothesis to explain sleep related erections was described in the article published by Bancroft (2005) who suggest that the noradrenergic neurons of the locus ceruleans are inhibitory to penile erection, and that the cessation of their discharge that occurs during REM sleep may allow testosterone-related excitatory actions to manifest as NPT [4].

The frequency of REM sleep in a nighttime correlates with the frequency of regular erections of an adult. In elderly, both the sleep architecture and the erectile reserve are modified, so the relationship between them is less remarkable [5,6].

Significant penile pain that occurs during erections related to REM sleep stage characterizes SRPE. According to the International Classification of Sleep Disorders [7], sleep-related painful erections are characterized by penile pain during sleep-related erections, typically during REM sleep stage, and was first described by Karacan in 1971. [8] Certainly, the rarity of the published cases undoubtedly does not reflect the actual occurrence of SRPE [9] (Figure 2).

As main characteristics of this condition is the presence of awakenings and unpleasant feelings of penile pressure or tension, or even unbearable pain. In those patients only the nocturnal erections are painful. Associated symptoms include sleep loss and daytime anxiety, tension and irritability.

The majority of the cases occur in men around 40 years of age with a chronic presentation and in severe forms with episodes of every night or more than once per night. SRPE tends to last longer than normal nocturnal 


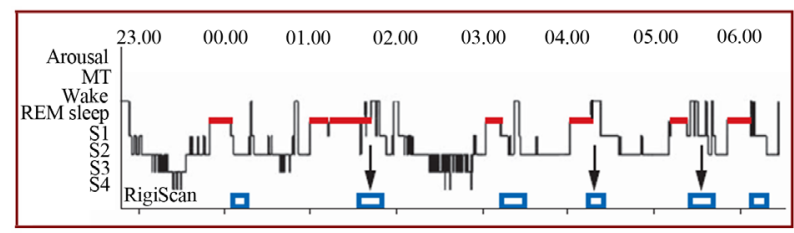

Figure 2. Polysomnography (hypnogram) coupled with penile tumescence recordings demonstrate three episodes of rapid eye movement sleep-related erection with pain and awakening. Black arrows show the three episodes of painful erections. Hypnogram (upper part of diagram): bold red lines represent REM sleep episodes; blue boxes represent nocturnal penile tumescence. Penile tumescence recording (lower part of diagram): shows an increase in diameter and rigidity at the tip and the base of the penis. The device consists of two loops placed around the tip and base of the penis. The values are given in $\mathrm{cm}$ and \% of lengthening of each loop. MT, movement time; REM, rapid eye movement; Rig, rigidity; S1 - S4, light to deep non-REM sleep; Tum, tumescence. Reprinted by permission from Macmillan Publishers (Karsenty et al. Sleep-related painful erections, nature clinical practice urology, may 2005 vol 2 no 5.)

erections. [10] The intensity of pain and the duration of the associated sleep loss increase during the second part of the night when REM sleep duration is longer. [10] Interesting is the fact that erections during sexual stimulation are painless. Patients usually report normal sexual activity with preserved libido and good frequency of intercourses [10].

Although the most frequently associated symptom was anxiety, psychiatric evaluation of the patients showed that sleep-related painful erections are not associated with any psychiatric diagnoses.

Ferini-Strambi et al. reported associated erectile dysfunction (impaired awake-state erections) in $23 \%$ of their patient series, but all of these patients had coexisting fully rigid nocturnal erection recordings. Such erectile dysfunction was most likely psychogenic, and related to sleep loss and anxiety. Anatomical abnormalities of the penis were always absent [11].

Etiologic hypotheses are scarce, ranging from autonomic nervous system disturbance with beta-adrenergic hyperactivity during sleep, to local disturbance of neurotransmission and endothelial function.

It is believed that SRPE may be a disorder of the autonomous nervous system occurring during sleep has been suggested by Ferrini-Strambi et al. [11] In their observations a reduced cardiac vagal activity and a possible beta-adrenergic hyperactivity during sleep was present. These aspects could be an explanation the transient response of SRPEs to beta-blockers [11].

Regarding the origin of pain, it has not been, until now, completely clarified.

Although not shown on EMG, it is thought to be due to spasms of the ischio and bulbocavernous muscles. The question that this diagnosis raises is the fact if surface electrodes are reliable in studying such spasms. Another possibility is ischemia of the cavernous blood, but unfortunately, no single case report reports cavernous blood gas analysis in SRPE [12].

Calvet et al. demonstrated bulbocavernous and ischiocavernous muscles hypertrophy on Doppler ultrasound in two of the three cases reported, suggesting that a disorder in the neurological control of these muscles may also explain SRPE [9].

No clear causative factors and no genetic predisposition have been found [6].

It is difficult to relate any other diagnosis with painful erections occurring exclusively during sleep. The most frequent differential diagnosis of SRPE are urethritis, Peyronie's disease, fimosis, high-flow priapism, lowflow priapism and metastasis to corpora cavernosa.

Phimosis and urethritis produce a clearly distinct clinical profile compared with sleep-related painful erections, with painful erection occurring during all erectile episodes. Peyronie's disease may also cause painful erection occurring during all erectile episodes, not only sleep related ones. Tumor metastasis to the corpora cavernosa is a very rare condition, often producing penile pain independent of erection. Bulky metastases can mimic permanent rigidity and also cause erosion of penile vessels leading to high flow priapism, but symptoms are independent of sleep, and clinical and sonographic examinations are abnormal. By definition, low-flow priapism lasts more than 4 hours, does not stop spontaneously, and is independent of sleep (except for a theoretical sickle cell anemia crisis triggered by hypoxemia during a sleep apnea period). Moreover, untreated or recurrent low-flow priapism leads to severe fibrotic damage of the corpora cavernosa and loss of erectile function, which was not the case of all reported patients until now as well as the current reported case.

As auxiliary exam for diagnosis, it is cited that polysomnography coupled with nocturnal penile tumescence recording may confirm this condition, documenting recurrent awakenings, mostly after REM sleep, accompanied by painful erections. Painless erections usually appear during periods of wakefulness and non-REM sleep [6].

However, the diagnosis of SRPE is possible supportedonly on clinical features, with no need of extensive complementary workup, whereas the most frequent differential diagnosis can be excluded with few, even none, additional exams. So SRPE, as a rare entity, must be managed as diagnosis of exclusion.

Most of therapeutic approaches are based on anecdotal reports. A variety of drugs have been attempted empirically, so far proved disappointing, as beta-adrenergics, tricyclics and antiandrogens.

The recently evidenced association between REM 
sleep and SRPEs raised the potential effect of substances witch suppress the REM state in inhibit the sleep-related erections.

As purposed by van Driel et al. [13], there are at least three pharmacologic approaches to treat SRPEs:

- Drug-induced blockage of erection

- Improvement of sleep architecture by suppressing REM sleep

- Suppression of pain or relaxation of pelvic floor muscles

Drugs directed to decrease erections, such as gosereline, leuproreline, bicalutamide, ketoconazole, estrogens, and others actually decrease sexual interest and are not considered appropriate to treat SRPEs, unless the patient is sexually inactive for other reason.

REM sleep may be suppressed by infinity of drugs, such as alcohol, monoamine oxidase inhibitors, tricyclic antidepressants, some selective serotonin reuptake inhibitors, CNS stimulants, and some hypnotic and sedative drugs, as benzodiazepines. Medications with prominent anti-cholinergic effects may also delay or suppress REM sleep.

Steigerand Benkert [14] described a long-term outcome after treatment using oral $25 \mathrm{mg}$ clozapine. Clozapine is an atypical anti-psychotic drug with noradrenergic, anticholinergic, antihistaminergic, and weak antidopaminergic properties, and may act by marked sedation and improvement of sleep architecture, with few adverse effects. [15]

The benzodiazepines (diazepan, bromazepan, clonazepan), with their REM sleep-suppressing properties, have been tested for treating SRPE by some authors [13] with success.

Clonazepan is a potent long action drug, initially used as anticonvulsive. It can maintain stable serum levels all night long, preventing recurrence of the painful erections.

Rourke (2006) [16] and van Driel (2008) [13] reported patients who have had good sustainable responses with oral baclofen at bedtime, in a 40mg oral dose. Traditionally prescribed to treat spasticity, the theoretical benefit consists in relaxation of pelvic muscles, specially bulbocavernous and ischiocavernous, acting in pain source.

In our reported case, it is noticed that the diagnosis has been made based on clinical aspects and complementary exams. Unfortunately, we did not documented the case with polysomnography since the patient was unable to proceed this evaluation by personal aspects and also the necessity of a prepared laboratory for this evaluation was not currently available. As described above to establish this diagnosis it is not necessary perform polysomnography since it is cost and time demanding, and can be done based in a clinical approach. Finally, the patient has had satisfactory response after the first therapeutic test and we judged not necessary perform this evaluation at this moment.

We believe that the excellent response to clonazepan associated with baclofen was achieved possibly because the improvement in sleep architecture and pelvis muscles relaxation. We reiterate that the patient suffered no impact on his sexual behavior and no other adverse effects have been claimed after this therapeutic intervention.

\section{Conclusions}

The sleep-related painful erections are certainly underdiagnosed. Many men are still resistant to express sexual problems, which can be evidenced by the chronic complaints with a mean delay between onset and diagnosis of months to years. Otherwise, clinicians usually do not ask about them and may not demand the needed attention when questioned by the patients.

Whether physiciancommunity and general population receive information about this clinical entity, more diagnoses will be suspected and the urologist will be the first referred physician for men presenting this problem, so he must know it. Moreover urologists must be aware about all sleep-related questions of their patients, whereas they have known negative influence in erectile function, beyond they can set out voiding difficulties.

After the first suspicion, polysomnography with rigidity measurements of nocturnal erections should be considered, although clinical diagnosis and therapeutic test may be acceptable.

Based on all literature reviewed, the initial treatment should safely consist in a REM sleep inhibitor (clonazepan), alone or coupled with baclofen. Other drugs should be reserved for failure of this first-line therapy. Drugs with impact on sexual desire and organic capacity for erections are not recommended.

\section{REFERENCES}

[1] I. Karacan, P. J. Salis, J. I. Thornby and R. L. Williams, "The Ontogeny of Nocturnal Penile Tumescence," Waking Sleeping, Vol. 1, 1976, pp. 27-44.

[2] I. Karacan, R. L. Williams, J. I. Thornby and P. J. Thalis, "Sleep Related Penile Tumescence as a Function of Age," American Journal of Psychiatry, Vol. 132, 1975, p. 932.

[3] C. Iber, S. Ancoli-Israel, A. Chesson and S. F. Quan, "The AASM Manual for the Scoring of Sleep and Associated Events: Rules, Terminology, and Technical Specification," American Academy of Sleep Medicine, Westchester, 2007.

[4] J. Bancroft, "The Endocrinology of Sexual Arousal," Journal of Endocrinology, Vol. 186, No. 3, 2005, pp. 411-427.

[5] M. Hirshkowit, C. A. Moore, C. R. Hamilton, et al., "Polysomnography of Adults and Elderly: Sleep Architecture, Respiration and Leg Movements,” Journal of Clinical Neurophysiology, Vol. 9, No. 1, 1992, p. 56. 
http://dx.doi.org/10.1097/00004691-199201000-00006

[6] G. Karsenty, E. Werth, P. A. Knapp, A. Curt, B. Schurch and C. L. Bassetti, "Sleep-Related Painful Erections," Nature Clinical Practice Urology, Vol. 2, No. 5, 2005, pp. 256-260. http://dx.doi.org/10.1038/ncpuro0184

[7] M. J. Thorpy, “Classification of Sleep Disorders,” Journal of Clinical Neurophysiology, Vol. 7, No. 1, 1990, pp. 67-81.

http://dx.doi.org/10.1097/00004691-199001000-00006

[8] I Karacan, "Painful Nocturnal Penile Erections," The Journal of the American Medical Association, Vol. 215, 1971, p. 1831.

[9] U. Calvet, "Painful Nocturnal Erection,” Sleep Medicine Reviews, Vol. 3, No. 1, 1999, pp. 47-57. http://dx.doi.org/10.1016/S1087-0792(99)90013-8

[10] A. Steiger and O. Benkert, "Examination and Treatment of Sleep Related Painful Erections. A Case Report,” Sleep Medicine Reviews, Vol. 18, No. 3, 1989, 263-267. http://dx.doi.org/10.1007/BF01543200

[11] L. Ferini-Strambi et al., "Sleep-Related Painful Erections: Clinical and Polysomnographic Features," Journal of Sleep Research, Vol. 5, No. 3, 1996, pp. 195-197.
http://dx.doi.org/10.1007/BF01543200

[12] B. J. Matthews and M. B. Crutchfield, "Painful Nocturnal Penile Erections Associated with Rapid Eye Movement Sleep,” Sleep, Vol. 10, No. 2, 1987, pp. 184-187.

[13] M. F. van Driel, J. J. Beck, H. W. Elzevier, J. H. van der Hoeven and J. M. Nijman, "The Treatment of Sleep-Related Painful Erections,” The Journal of Sexual Medicine, Vol. 5, No. 4, 2008, pp. 909-918. http://dx.doi.org/10.1111/j.1743-6109.2007.00645.x

[14] A. Steiger and O. Benkert, "Examination and Treatment of Sleep Related Painful Erections. A Case Report,” A Archives of Sexual Behavior, Vol. 18, No. 3, 1989, 263267. http://dx.doi.org/10.1007/BF01543200

[15] A. V. Cordioli e cols, Psicofármacos, Artmed, 3th ed., 2005.

[16] K. F. Rourke, A. H. Fischler and G. H. Jordan, “Treatment of Recurrent Idiopathic Priapism with Oral Baclofen,” The Journal of Urology, Vol. 168, No. 6, 2002, pp. 2552-2553. http://dx.doi.org/10.1016/S0022-5347(05)64201-2 